\title{
Unique aspects of pain reduction in osteoarthritis of the knee with LMWF-5A
}

This article was published in the following Dove Press journal:

Open Access Rheumatology: Research and Reviews

10 February 2015

Number of times this article has been viewed

\section{Brian McGrath \\ University Orthopaedic Center, Amherst, NY, USA}

Correspondence: Brian McGrath University Orthopaedic Center, 4949 Harlem Road, Amherst, NY 14226, USA

Tel +I 7I6 2043217

$\mathrm{Fax}+\mathrm{I} 7162043283$

Email bem4@buffalo.edu
Abstract: Osteoarthritis of the knee (OAK) is a common form of arthritis that can lead to substantial pain and disability. This commentary highlights key aspects of the recently published phase 3 A Efficacy and Safety Study of Two Doses of Intra-Articular Injection of Ampion $^{\mathrm{TM}}$ in Adults With Pain Due to Osteoarthritis of the Knee (SPRING) study. SPRING (NCT01839331) was a multicenter, randomized, vehicle-controlled, double-blind trial that evaluated the safety and efficacy of the low-molecular-weight fraction of 5\% human serum albumin (LMWF-5A) for treatment of pain, measured by the Western Ontario and McMaster Universities Osteoarthritis (WOMAC) pain scale, in patients with symptomatic OAK (N=329). Patients in this study reflected many characteristics of "real-world" individuals with OAK, with a broad range of disease severity and disability. The most important finding from this study was that treatment with a single intra-articular injection of LMWF-5A led to significant pain reduction in the patients with objective radiographic evidence of severe disease and joint deterioration (Kellgren-Lawrence grade 3; $P=0.04$ and Kellgren-Lawrence grade 4; $P=0.02$ ). The magnitude of pain reduction in the entire cohort treated with LMWF-5A was $42 \%$ from baseline and the treatment effect compared with vehicle control (estimated difference in WOMAC pain, -0.25 ; $P=0.004)$ was also notable, especially relative to a previously reported study of hyaluronic acid, in which only a marginally significant treatment effect was observed (mean difference in WOMAC pain compared with control, $-0.15 ; P=0.047)$. Significant improvement in physical function observed with LMWF-5A $(P=0.04)$ was also noted and suggests that LMWF-5A may provide therapeutic benefit for those who are limited in the activities of daily living. Intra-articular injection of LMWF-5A was well tolerated, and the adverse event profile was similar to that of control. These results demonstrate significant benefit of LMWF-5A for patients with greatest disability (Kellgren-Lawrence grades 3 and 4) and highest therapeutic need who have limited pharmacologic options for relief of pain associated with OAK.

Keywords: human serum albumin, knee, osteoarthritis, pain, Kellgren-Lawrence

\section{Introduction}

Osteoarthritis is the most common form of arthritis and affects $\sim 27$ million people in the United States. ${ }^{1}$ Osteoarthritis of the knee (OAK) occurs in $\sim 12 \%$ of individuals older than 60 years, ${ }^{1,2}$ and $\sim 60 \%$ of these patients may have moderate-to-severe OAK, which is associated with limitations in daily activities and loss of productivity. ${ }^{3}$ Osteoarthritis is usually a progressive disease that develops from failed repair of joint damage resulting from stresses that may originate from an abnormality in any of the synovial joint tissues. ${ }^{4}$ Pathological changes leading to the breakdown of cartilage and bone eventually result in substantial pain and disability. ${ }^{3,4}$ It has been proposed that 
osteoarthritis is not a single disease but a heterogeneous collection of subtypes, which can be characterized by joint inflammation. ${ }^{4}$

Current therapies for pain reduction in OAK include physical activity, weight loss, acetaminophen, nonsteroidal antiinflammatory drugs, intra-articular steroids, and hyaluronic acid (HA). ${ }^{5}$ Patients with pain uncontrolled by these treatments may eventually be eligible for knee surgery or replacement. ${ }^{6}$ Treatments such as intra-articular steroids can provide short-term pain reduction but may lose effectiveness over time and may not lead to improvements in physical function or stiffness. ${ }^{5}$ As the American Association of Orthopaedic Surgeons clinical practice guidelines could not recommend use of intra-articular steroids or $\mathrm{HA},{ }^{7}$ there is a need for an agent that would halt or reverse disease progression and provide antiinflammatory and analgesic benefits, especially for treatment of OAK in aging or obese patients. ${ }^{8}$ Burden of disease is especially high in the latter population, as it is estimated that OAK is associated with 1.86 quality-adjusted life-years lost for nonobese patients aged 50 to 84 years versus 3.50 for obese patients. ${ }^{9}$

Human serum albumin has been approved for treatment of burns and shock for more than 30 years, with favorable safety and tolerability. ${ }^{8,10}$ The low-molecular-weight fraction $(<5,000 \mathrm{Da})$ of $5 \%$ human serum albumin (LMWF-5A) contains aspartyl-alanyl diketopiperazine, which may have antiinflammatory or immunomodulatory effects. ${ }^{8,11}$ Primary results from the SPRING study of LMWF-5A for pain reduction in OAK were recently published. ${ }^{8}$ This commentary highlights the unique aspects of this study of LMWF-5A in a heterogeneous "real-world" cohort of patients with moderate to severe OAK.

\section{Commentary main text}

\section{Study design and methods}

A Efficacy and Safety Study of Two Doses of Intra-Articular Injection of Ampion ${ }^{\mathrm{TM}}$ in Adults With Pain Due to Osteoarthritis of the Knee (SPRING) study (NCT01839331) was a multicenter, randomized, vehicle-controlled, double-blind trial to evaluate the safety and efficacy of LMWF-5A for treatment of pain in symptomatic OAK. ${ }^{8}$ Patients $(\mathrm{N}=329)$ were randomized to receive a 4 or $10 \mathrm{~mL}$ intra-articular injection of LMWF-5A or vehicle control (saline). At baseline, mean age of patients was $\sim 62$ years (range, $41-84$ years); and $64 \%$ were female. ${ }^{8}$ Patients in SPRING reflected many characteristics of individuals with OAK that may not have been represented in previous clinical studies, ${ }^{12,13}$ including greater ethnic diversity ( $9 \%$ were nonwhite) and increased body mass index (mean, $\left.33 \mathrm{~kg} / \mathrm{m}^{2}\right){ }^{8}$
The primary endpoint in SPRING was difference in pain reduction between LMWF-5A and control, measured by change from baseline in Western Ontario and McMaster Universities Osteoarthritis (WOMAC) A subscore at 12 weeks. ${ }^{8}$ Secondary endpoints included changes in physical function (WOMAC C) and pain with movement and at rest (WOMAC A). Safety assessments included incidence and severity of adverse events (AEs) ${ }^{8}$

\section{Efficacy and safety}

Nearly two-thirds of patients enrolled in SPRING had moderate (Kellgren-Lawrence [K-L] grade 3, 42\%) or severe (K-L grade $4,22 \%$ ) symptomatic OAK, ${ }^{8,14}$ which differs from a pivotal trial of high-molecular-weight $\mathrm{HA}$ in patients with milder disease (K-L grades 2 and 3 only; K-L grade 4 was excluded).${ }^{13}$ Patients with more severe disease are more likely to demonstrate joint deterioration that may be assessed more accurately. ${ }^{15,16}$

The most important result in SPRING was the statistically significant pain reduction in patients with $\mathrm{K}$-L grade $3(P=0.04)$ and grade 4 (estimated treatment difference in WOMAC pain with LMWF-5A relative to control, $-0.42 ; P=0.02) .{ }^{8}$ These results are noteworthy, given that this population has the greatest degree of joint damage, and significant effects have not been consistently demonstrated using other agents. For example, Chevalier et al reported that a single injection of HA led to a $31.3 \%$ pain reduction from baseline at 26 weeks. ${ }^{13}$ The effect observed with HA did not exceed a clinically meaningful threshold $(-40.8 \%)^{16}$ and was associated with only a marginally significant difference compared with control $(-0.15 ; P=0.047)$ in patients with less severe disease. ${ }^{12,13}$ Despite approval, the American Association of Orthopaedic Surgeons could not recommend HA for treatment of OAK. ${ }^{7}$

In SPRING, improvements in pain were observed with LMWF-5A in the overall cohort and across most subgroups. ${ }^{8}$ Significant clinical efficacy for pain reduction with LMWF-5A relative to control was observed as early as week $4(P=0.03),{ }^{8}$ an observation that has not been consistently demonstrated in previous studies of pain relief in OAK. ${ }^{12,13,17}$ Notable aspects were the clinically relevant ${ }^{16}$ magnitude of pain reduction ( $42 \%$ from baseline) and the significant effect compared with control (treatment difference, -0.25 ; $P=0.004$ ) observed with a single injection of LMWF-5A. ${ }^{8}$

Improvement in physical function (WOMAC $\mathrm{C}$; $P=0.04$ ) was also demonstrated, suggesting LMWF$5 \mathrm{~A}$ can provide therapeutic benefit in patients who may have substantial functional impairment. ${ }^{8}$ Moreover, the WOMAC C subscore may not capture all 
aspects of improved function (eg, increased activity) that may positively affect quality of life for many patients.

Severity of OAK also may have influenced the treatment effects in this study, as a more pronounced placebo effect was observed in patients with milder disease (K-L grade 2), potentially leading to nonsignificant differences between LMWF-5A and control in this subgroup. Placebo effects are often observed in clinical studies of osteoarthritis, ${ }^{18}$ however, in SPRING, there was a consistent separation between treatment groups in patients with moderate-to-severe OAK (K-L grades 3 and 4), validating these results in a subgroup with objective radiographic evidence of joint damage. ${ }^{8}$

The overall safety profile of LMWF-5A was acceptable, and no serious drug-related AEs occurred. The percentage of AEs reported was similar in patients who received LMWF-5A (41\%) or saline $(47 \%){ }^{8}$

Potential limitations of the SPRING study include the relatively short follow-up time (12 weeks), which may not have captured the maximum difference between treatment groups. In addition, as efficacy measures could be affected by pain in the nontreated knee, bilateral disease was not evaluated in SPRING. Therefore, it was not possible to determine whether the treatment effect differed between patients with unilateral versus bilateral OAK. A planned phase 3 study with a longer observation period may assess the maximum treatment effect of LMWF-5A in OAK.

\section{Conclusion}

Results from the SPRING study exceeded expectations, given that a majority of patients enrolled had moderate to severe symptomatic OAK. There was a significant benefit of LMWF-5A for patients with objective radiographic evidence of OAK who have limited pharmacologic treatment options for pain associated with this disease.

\section{Acknowledgments}

This work was supported by Ampio Pharmaceuticals, Inc. Editorial support in the form of developing the commentary outline and first draft, suggesting editorial revisions to drafts, collating author comments, copyediting, fact checking, and referencing was provided by Rebecca E. Slager and Jennifer Rossi at MedThink SciCom and was funded by Ampio Pharmaceuticals, Inc.

\section{Disclosure}

$\mathrm{BM}$ is a consultant for Ampio Pharmaceuticals, Inc. The author reports no other conflicts of interest in this work.

\section{References}

1. Dillon CF, Rasch EK, Gu Q, Hirsch R. Prevalence of knee osteoarthritis in the United States: arthritis data from the Third National Health and Nutrition Examination Survey 1991-1994. J Rheumatol. 2006;33(11): 2271-2279.

2. Lawrence RC, Felson DT, Helmick CG, et al; National Arthritis Data Workgroup. Estimates of the prevalence of arthritis and other rheumatic conditions in the United States. Part II. Arthritis Rheum. 2008;58(1): 26-35.

3. Sadosky AB, Bushmakin AG, Cappelleri JC, Lionberger DR Relationship between patient-reported disease severity in osteoarthritis and self-reported pain, function and work productivity. Arthritis Res Ther. 2010;12(4):R162.

4. Lane NE, Brandt K, Hawker G, et al. OARSI-FDA initiative: defining the disease state of osteoarthritis. Osteoarthritis Cartilage. 2011;19(5): $478-482$.

5. Zhang W, Nuki G, Moskowitz RW, et al. OARSI recommendations for the management of hip and knee osteoarthritis: part III: Changes in evidence following systematic cumulative update of research published through January 2009. Osteoarthritis Cartilage. 2010;18(4): 476-499.

6. Quintana JM, Arostegui I, Escobar A, Azkarate J, Goenaga JI, Lafuente I. Prevalence of knee and hip osteoarthritis and the appropriateness of joint replacement in an older population. Arch Intern Med. 2008;168(14):1576-1584.

7. American Academy of Orthopaedic Surgeons. Treatment of Osteoarthritis of the Knee. Evidence-Based Guideline. 2nd edition. 2013. Available from: http://www.aaos.org/Research/guidelines/TreatmentofOsteoarth ritisoftheKneeGuideline.pdf. Accessed July 21, 2014.

8. Bar-Or D, Salottolo KM, Loose $\mathrm{H}$, et al. A randomized clinical trial to evaluate two doses of an intra-articular injection of LMWF-5A in adults with pain due to osteoarthritis of the knee. PLoS One. 2014;9(2): e87910.

9. Losina E, Walensky RP, Reichmann WM, et al. Impact of obesity and knee osteoarthritis on morbidity and mortality in older Americans. Ann Intern Med. 2011;154(4):217-226.

10. Quinlan GJ, Martin GS, Evans TW. Albumin: biochemical properties and therapeutic potential. Hepatology. 2005;41(6):1211-1219.

11. Bar-Or D, Thomas GW, Bar-Or R, et al. Commercial human albumin preparations for clinical use are immunosuppressive in vitro. Crit Care Med. 2006;34(6):1707-1712.

12. Synvisc-One ${ }^{\sqrt{ }}$ [package insert]. Ridgefield, NJ: Genzyme Biosurgery; 2010.

13. Chevalier X, Jerosch J, Goupille P, et al. Single, intra-articular treatment with $6 \mathrm{ml}$ hylan G-F 20 in patients with symptomatic primary osteoarthritis of the knee: a randomised, multicentre, double-blind, placebo controlled trial. Ann Rheum Dis. 2010;69(1): 113-119.

14. Kellgren JH, Lawrence JS. Radiological assessment of osteo-arthrosis. Ann Rheum Dis. 1957;16(4):494-502.

15. Braun HJ, Gold GE. Diagnosis of osteoarthritis: imaging. Bone. 2012;51(2):278-288.

16. Tubach F, Ravaud P, Baron G, et al. Evaluation of clinically relevant changes in patient reported outcomes in knee and hip osteoarthritis: the minimal clinically important improvement. Ann Rheum Dis. 2005;64(1): 29-33.

17. Synvisc ${ }^{\circledR}$ [package insert]. Ridgefield, NJ: Genzyme Biosurgery; 2010.

18. Zhang W, Robertson J, Jones AC, Dieppe PA, Doherty M. The placebo effect and its determinants in osteoarthritis: meta-analysis of randomised controlled trials. Ann Rheum Dis. 2008;67(12):1716-1723. 


\section{Publish your work in this journal}

Open Access Rheumatology Research and Reviews is an international, peer-reviewed, open access journal, publishing all aspects of clinical and experimental rheumatology in the clinic and laboratory including the following topics: Pathology, pathophysiology of rheumatological diseases; Investigation, treatment and management of rheumatological

diseases; Clinical trials and novel pharmacological approaches for the treatment of rheumatological disorders. The manuscript management system is completely online and includes a very quick and fair peerreview system, which is all easy to use. Visit http://www.dovepress.com/ testimonials.php to read real quotes from published authors.

Submit your manuscript here: http://www.dovepress.com/open-access-rheumatology-research-and-reviews-journal 